\section{The promise of microbial engineering for developing new strategies for tackling human disease}

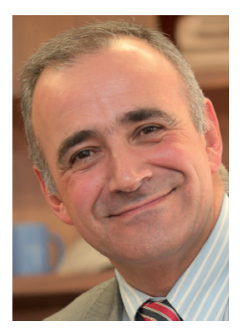

\author{
“...the synthetic manipulation of bacteria and \\ viruses allows the design of new strategies that \\ offer both hope and new opportunities in the fight \\ against human diseases."
}

Ignacio López-Goñi

Department of Microbiology \& Parasitology, Institute of Tropical Health, University of Navarra, 31008 Pamplona, Spain

Tel.: +34948425600 milgoni@unav.es

In May 2010 a team lead by J Craig Venter reported the creation of a bacterial chromosome and its transfer into a bacterium where it successfully replicated [1]. This work demonstrated the design, synthesis and assembly of a synthetic genome of Mycoplasma mycoides and its subsequent transfer into Mycoplasma capricolumas, a receptor cell, to create a new strain of $M$. mycoides that was controlled by a synthetic chromosome. The bacterium was able to self-replicate and synthesize new proteins. It took 15 years for this work to be completed, during which time new tools, techniques and concepts from synthetic biology were developed. This demonstrates the enormous possibilities for the future offered by microbial engineering, the discipline combining microbiology, molecular biology and chemical engineering; that is, the manipulation of microorganisms for the development of new products and applications for use in humans.

\section{"This demonstrates the enormous possibilities for the future offered by microbial engineering, the discipline combining microbiology, molecular biology and chemical engineering..."}

In recent months, two studies have been published that, owing to their originality and underlying strategy, are two new pieces of evidence showing the future possibilities of manipulating microorganisms, in both cases to combat human diseases. In one study, bacteria have been manipulated to fight against infectious disease, and in the other, viruses have been modified as a tool to combat cancer.
One of the studies, published in Molecular Systems Biology [2], is based on synthetic biology techniques by which a microbe is manipulated so that it performs functions that it is normally unable to carry out, as is the case of killing another bacterium, to prevent or treat Pseudomonas aeruginosa infections in humans. The lack of progress in the development of new antibiotics, together with the increase in the emergence of multiresistant pathogens make the development of new antimicrobial strategies a priority. Saeidi et al. [2] have designed a novel microbial engineering system that allows P. aeruginosa, a Gram-negative bacterium capable of colonizing the respiratory and gastrointestinal systems in humans, to be detected and killed. $P$. aeruginosa is the cause of approximately $10 \%$ of all nosocomial infections and is especially serious in immunocompromised patients. Normally, such infections are treated with antibiotics, but $P$. aeruginosa is intrinsically resistant to many antibiotics and antimicrobials, in part owing to the efficacy of its efflux-type systems. In this study, the authors describe the design and construction of a genetic system using, as a vehicle, a nonpathogenic strain of Escherichia coli capable of detecting the $P$. aeruginosa pathogen, exploding and then releasing antimicrobial substances which kill Pseudomonas - a system reminiscent of a real bacterial 'kamikaze'.

This new design, called the pathogen sensing and killing system, functions in various stages. First, the bacterial 'kamikaze' (E. coli) detects the molecules of acyl homoserine lactone produced by the quorum sensing system of Pseudomonas; then in E. coli the production of

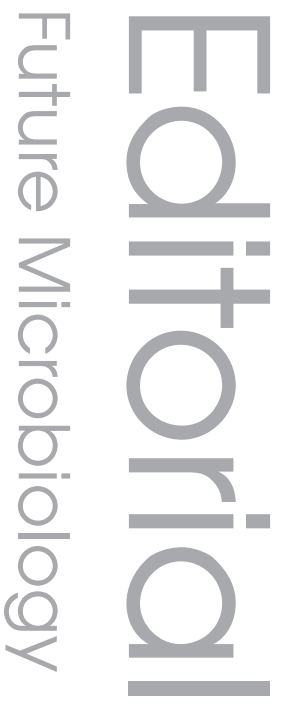

\section{Keywords}

- microbial engineering

- oncolytic virus

- pseudomonas - pyocins

- quorum-sensing

- synthetic biology = viral

therapy

Future : Medicine part of 
a toxic protein, pyocin S5, is activated together with the action of another E7 protein, which causes the E. coli to explode and to lyze. In this way the pyocin S5 is released into the external medium, which kills Pseudomonas (unlike what happens with traditional antibiotics, resistance to pyocins is infrequent). Following this strategy, the E. coli release the toxic pyocin only in the presence of Pseudomonas. The pyocin does not affect other microbes, which means that this treatment should not kill other bacteria that are beneficial to the organism. Furthermore, the researchers have demonstrated that this system not only works when Pseudomonas is growing freely in liquid culture media, but also when the bacterium forms biofilms when it multiplies inside the organisms. It has still not been tried in experimental infections in animals but if it were found to work, one possibility would be to administer the treatment as a probiotic drink to immunocompromised patients to prevent Pseudomonas infections.

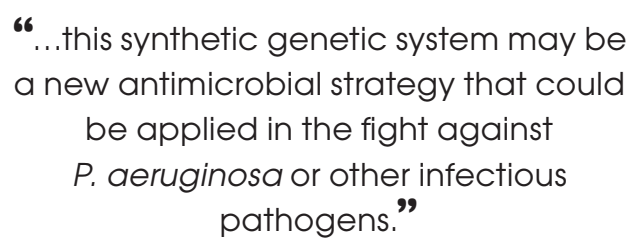

The results suggest that this synthetic genetic system may be a new antimicrobial strategy that could be applied in the fight against $P$. aeruginosa or other infectious pathogens. It is also possible that this mechanism could be modified to become active against other similar molecules produced by the quorum sensing systems of other pathogens, such as Vibrio cholera or Helicobacter pylori.

Another of the recent strategies is based upon the manipulation of a virus. Imagine that we were capable of developing a vehicle that was sufficiently intelligent to selectively seek out cancer cells, without affecting the remaining healthy cells, and inject them with an antidote that would lead to their death - a real 'magic bullet' against cancer. This is, more or less, what a group of researchers have achieved, as published in a recent issue of Nature [3]. To this end, the researchers genetically modified the vaccinia virus, well-known for its use over many years in the eradication of smallpox. They designed a new strain of the virus, called JX-594, so that it was able to replicate, express a foreign gene and amplify in tumor cells. JX-594 is an oncolytic virus designed to seek out, attack and destroy tumor cells. It is designed to reproduce selectively in tumor cells that exhibit activity via EGFR/Ras, but not in normal tissue. In this study the authors conducted a clinical trial on a group of patients all of whom had advanced cancer affecting various organs and who did not respond to conventional treatments. The patients received a single injection of the genetically modified JX-594 virus at different doses. Analysis of the biopsies from the tumors showed that in several patients the virus was able to infect and multiply in tumor cells but not in normal healthy tissue. Furthermore, expression of the foreign gene that had been inserted into the virus to aid its detection was also discovered in the tumors. This virus was well tolerated by the patients even at the highest doses as the only side effect was a flu-like malaise, which lasted only 1 day.

This is the first time that a viral therapy that replicates exclusively in tumoral tissue after intravenous injection has been successful in humans. This strategy allows foreign genes to be selectively expressed in tumor cells, which opens up the possibility of selectively inserting therapeutic substances into solid tumors in humans. Although with this approach the researchers did not manage to cure any of the patients, they have shown that we are already able to modify certain viruses, inject them into patients, make the virus seek out only tumor cells while ignoring all healthy cells and have them inject the appropriate antidote. The authors of this paper have produced the 'magic bullet', which perhaps in the future we may tailor to make it effective against different types of cancer.

\section{"This strategy allows foreign genes to be selectively expressed in tumor cells, which opens up the possibility of selectively inserting therapeutic substances into solid tumors in humans."}

Curing human diseases is a complex problem that requires complex solutions. It is only through coordinated and interdisciplinary work that teams of microbiologists, molecular biologists, computer biologists, engineers, chemists, physicians and other professionals will be able to provide new and original solutions. In recent months we have seen examples of how the synthetic manipulation of bacteria and viruses allows the design of new strategies that offer 
both hope and new opportunities in the fight against human diseases.

\section{Financial \& competing interests disclosure}

The author has no relevant affliations or financial involvement with any organization or entity with a financial interest in or financial conflict with the subject matter or materials discussed in the manuscript. This includes employment, consultancies, honoraria, stock ownership or options, expert testimony, grants or patents received or pending, or royalties.

No writing assistance was utilized in the production of this manuscript.

\section{References}

1. Gibson DG, Glass JI, Lartigue C et al. Creation of a bacterial cell controlled by a chemically synthesized genome. Science 329(5987), 52-56 (2010).

2. Saeidi N, Wong CK, Lo TM et al. Engineering microbes to sense and eradicate Pseudomonas aeruginosa, a human pathogen. Mol. Syst. Biol. 7, 521 (2011).

3. Breitbach CJ, Burke J, Jonker D et al. Intravenous delivery of a multi-mechanistic cancer-targeted oncolytic poxvirus in humans. Nature 477(7362), 99-102 (2011). 